
rized by a significant energy-consuming system during the crushing workflow. The current trend in the development of such processes puts forward requirements for the development of new or improvement of existing energy-saving equipment. The essence of the solution to the problem in this work is determined by using resonant modes, which are inherently the most effective. The practical implementation of the resonance mode has been achieved taking into account the conditions for the interaction of the resonant vibration crusher with the material at the stages of its destruction. The degree of the stress-strain state of the material is taken into account, which was a prerequisite for identifying the potential for the development of a vibration load. Composed equations of motion based on a substantiated discrete-continuous model of a vibration crusher and processing material. An approach is applied to determine the steproise destruction of the material with the determination of the required degree of energy. This methodological approach made it possible to reveal the nature of the process of material destruction, where energy costs at the stages of crack formation, their development and final destruction are taken into account. It was revealed that the greatest energy consumption during the operation of crushers goes into the kinetic energy of the crushing plates and the potential energy of deformation of the springs. The proposed model is common for any design of a vibration machine and its operating modes. The stable resonance mode has made it possible to significantly reduce the energy consumption for the course of the technological process of material grinding. The results obtained are used to improve the calculation methods for vibratory jaw and cone crushers that implement the corresponding energy-saving stable zones of the working process

Keywords: vibration crusher, crushing chamber, resonant mode, process of destruction, energy, stress, deformation
UDC 621.926.22

DOI: $10.15587 / 1729-4061.2021 .239292$

\section{DETERMIANTION OF ENERGY CHARACTERISTICS OF MATERIAL DESTRUCTION IN THE CRUSHING CHAMBER OF THE VIBRATION CRUSHER}

I van $\mathbf{N}$ azare n k o

Corresponding author

Doctor of Technical Sciences, Professor, Head of Department*

E-mail: i_nazar@i.ua

Yevhen Mishchuk

PhD, Associate Professor*

D mitry Mishch uk

$\mathrm{PhD}$, Associate Professor

Department of Construction Vehicles**

Mykola Ruchynskyi

PhD, Professor*

Ivan Rogovski i

Doctor of Technical Sciences, Senior Researcher Research Institute of Engineering and Technology****

Li udm y la Mikhailova PhD, Professor

Department of Electrical Engineering,

Electromechanics and Electrotechnology

Educational and Scientific Institute of Energy State Agrarian and Engineering University in Podilia Shevchenko str., 13, Kamianets-Podilsky, Ukraine, 32300

Li ud m y la Titova PhD, Associate Professor*** Mykola Berezovyi

$\mathrm{PhD}$, Associate Professor Department of Mechanics****

Ruslan Shatrov

$\mathrm{PhD}$, Associate Professor*** *Department of Vehicles and Equipment of Technological Processes** **Kyiv National University of Construction and Architecture Povitroflotskyi ave., 31, Kyiv, Ukraine, 03037 ***Department of Technical Service and Engineering Management named after M. P. Momotenko**** $\star * \star *$ National University of Life and Environmental Sciences of Ukraine Heroiv Oborony str., 15, Kyiv, Ukraine, 03041
Received date 14.06.2021 Accepted date 20.08.2021 Published date 31.08.2021
How to Cite: Nazarenko, I., Mishchuk, Y., Mishchuk, D., Ruchynsky, M., Rogovskii, I., Mikhailova, L., Titova, L., Berezovyi, M., Shatrov, R. (2021). Determiantion of energy characteristics of material destruction in the crushing chamber of the vibration crusher. Eastern-European Journal of Enterprise Technologies, 4 (7 (112)), 41-49. doi: https://doi.org/10.15587/17294061.2021 .239292
1. Introduction

The construction industry is one of the leading industries worldwide. Thus, the share of construction work in the total volume of world GDP is today about $13 \%$ and continues to grow every year. An increase in the volume of construction work cannot but lead to an increase in energy consumption for the production of building materials. On the other hand, 
the rate of development of scientific and technological progress is growing every year. In this case, this leads to increased requirements for the quality of construction work and materials, as well as the introduction of new methods and approaches to the implementation of construction work.

A significant part of the energy consumption in the production of building materials is spent on the crushing or grinding process. The main equipment used for crushing materials is crushers. Therefore, one of the methods to reduce energy consumption for the production of a unit of building material is to increase the energy efficiency of crushing machines.

In general, all approaches to improving the energy efficiency of a crushing machine can be divided into three categories:

1) development of a new design;

2) improvement of the existing design;

3) optimization of the workflow.

It is advisable to consider the methodology for solving the problem, which belongs to the third category, since the optimization of the workflow allows to propose those modes and parameters that will ensure the energy saving of the material crushing process. The working process of any crusher is the destruction of the material in the crushing chamber. The energy that is spent on the execution of the working process consists of the energy of the movement of the elements of the machine and the energy of the destruction process. The process of destruction of material in the crushing chamber is a rather complex process, which is influenced by a number of different factors that have a probabilistic character. The main factors include the shape of the material and its strength, the presence of cracks in the material (the size of the crack, its dislocation relative to the applied force and the number). It is also necessary add the location of the material itself in the crushing chamber (resting on the working surfaces of the machine or interacting with other pieces of crushing material), etc.

Thus, an urgent task, in addition to creating new crushing equipment, is the task of reducing the energy consumption of the existing crushing equipment.

\section{Literature review and problem statement}

In [1], the regularities of crack propagation on the surface of a turbine blade are investigated using the Ansys package (USA). The process of blade failure in the presence of a crack is considered based on the use of two parameters of the stress intensity factor and the J-integral. In general, the problem is solved by the finite element method using the Crack object and Fracture Tool packages built into Ansys. The advantages of this modeling method are the flexibility and speed of modeling objects of the same type, however, it does not have a clear understanding of the mathematical apparatus that is used by the program to solve the problem. In addition, it is not clear how the program takes into account the change in the stress intensity factors and the J-integral when simulating the volumetric propagation of a crack.

The study of the energy of destruction of a rock mass based on the improved dependence of Griffiths is considered in work [2]. The dependence on the determination of the energy of destruction contains the parameter of the critical crack length and the corrected parameter of the specific surface energy based on the regression analysis of the experimental data. This dependence is somewhat difficult to use, since it includes the critical crack length and specific surface energy, the determination of which is not an easy task. On the other hand, this dependence is suitable for use when considering the problem of destruction only on a plane.

In the source [3], the author argues that the growth of the crack and the destruction of the material occur along the shear lines, that is, the forces of contact friction play a key role in the destruction. However, using these dependencies to optimize the crushing process is difficult because they include the geometric characteristics of the fracture. In addition, shock loads should be taken into account when vibrating the material [4]. When exposed to shock loads, chips occur in the material due to the passage of the shock wave. In this case, shear stresses may not play a key role in crack propagation.

In work [5], the fracture energy is proposed to be determined on the basis of the dependence of J. Swensen and J. Murkes, which is based on the hypothesis of F. Bond. By correlating the dependence of J. Swensen and J. Murkes with the consumption of electricity, the author deduces the parameter of constant current strength, necessary to reduce the particle size, is destroyed. As it is known, the Bond hypothesis is best suited to describe the crushing process in the middle range of particle size. Thus, the description of the crushing process in [5] is given for a narrow range of crushing machines. The classical hypothesis of energy consumption for the destruction process (hypothesis of volumes) is used in [6] to calculate the critical speed of material ejection from the rotor of a centrifugal impact crusher. This hypothesis is best suited for describing the energy consumption for coarse crushing [7]. The hypotheses given in the sources [5,6] do not take into account the presence of breaks in the material structure in the form of a crack.

In [8], the magnitude of the normal and landslide compliance of a three-dimensional crack is calculated, taking into account the contact between the shores. Compliance is the relative movement of points to which a load is applied. At the same time, there is no experimental confirmation of these results. In [9], studies of crack propagation in reference specimens made of quartz sand and high strength bassanite (calcium sulfate hemihydrate) are presented. However, the use of exclusively static loads limits practical use. This is due to the fact that in most crushing machines, dynamic loads also arise next to static ones. In [10], the modeling of the process of crushing the material of a jaw crusher is considered. The software Rocky (USA) is used for modeling, designed to simulate technological processes of bulk materials. Thus, the computational model of the material is discrete. To determine the granulometric composition of the crushing products, the work uses the formulas of A. Gaudin and A. Rosen-E. Rammler. In addition, it is noted that the results of the experiment on crushing single pieces of material may be inaccurate, which is due to the failure to take into account the continual model of the material. The work [11] investigates the dynamic destruction of material in a vibratory jaw crusher. To determine the specific energy consumption, dependence is used, which is built on a combination of the theory of volumes and the theory of surfaces. A brittle material is used as a working fluid, as exemplified by sandstone, and a plastic material, as exemplified by talc. This work is based on energy hypotheses that do not take into account the presence of a crack in the material, and the fracture process itself is considered as a single process.

The above methods for determining the energy of destruction of a material differ significantly from each other, based on various energy hypotheses, both according to the accepted assumptions and according to the results obtained. 
Thus, the lack of a generally accepted model of the grinding process and a unified methodology for determining the energy consumption of the process of destruction of materials of crushing machines is the problem that needs to be addressed.

\section{The and objectives of research}

The aim of research is to determine the energy costs for the process of material destruction in the crushing chamber of a vibratory jaw crusher. This will enable the development of energy-saving and efficient grinding equipment.

To achieve this aim, the following objectives are identified:

- to investigate and analyze changes in the main parameters affecting the zones to ensure the reduction of energy consumption of the vibration crusher;

- to determine the modes and rational parameters to ensure a decrease in energy consumption and an increase in the efficiency of the technological process of material grinding.

\section{Materials and methods for the study of energy costs}

The study of the process of material destruction and the determination of energy consumption is based on the basic principles of the mechanics of fracture of solids, the generally accepted principles of the theory of strength, the theory of mechanical vibrations and the theory of continuous media.

The mathematical model of the «crusher - material» vibration system is based on the following assumptions. The material to be destroyed behaves like an absolutely elastic body and is isotropic. The shape of the material to be destroyed is assumed to be spherical. First, a solid spherical body is considered to which of two opposite sides an external force is applied from the action of crushing plates. A spherical coordinate system is taken as the main computational system. Next, a system of equations is written that determines the movement of the body in three coordinates. Volumetric spherical functions are used to solve these equations. Then the corresponding displacements and stresses are found. Next, let's consider a body that contains a crack at the center of coordinates. The crack shape is ellipsoid. Then the displacements on the crack surface from the action of tensile forces in elliptical coordinates are determined. Further, based on the superposition of two stress states (a body without breaking the integrity and a body with a crack), their displacements are summed up. Thus, solutions to the problem of fracture of a body in which there is a crack are obtained.

The structural elements of the machine take an elementary form, the gravitational forces of individual links are applied at certain points, the forces of resistance to destruction are presented in the form of a total force that is applied to the center of the cheek. When compiling the equations of motion of vibrational systems, Hamilton's variational principle is used. When calculating the parameters of a vibrating jaw crusher, the crushing material is taken into account by adding to the corresponding masses of the system interacting with the working material, the coefficient of added mass multiplied by the total mass of the material. At the final stage of the study, the total energy consumption of vibration crushers is determined taking into account the stress state of the material with a crack. To perform mathematical operations in solving the problem, the software Wolfram Mathematica 11.3 (USA) was used. When calculating a cone crusher, the material is taken in the form of the Voigt model, it was successfully used in works [12,13] using a discrete model [14].

Based on the Poincaré hypothesis [15], according to the generalized physical model of the shape of the material, one can take the sphere. The main provisions and the final proof of the Poincaré conjecture are presented in [16].

All possible contacts are reduced to several models [17]:

a) contact at two points;

b) contact at three points;

c) contact by point and area;

d) contact in two areas.

To determine the stressed and deformed state of a body under the influence of an external force on it and in the presence of a crack in it, the following method can be used [18]. The idea of the method is to apply the principle of superposition of solutions to two separate problems, namely:

1) the problem of the load is applied to the solid material of destruction;

2) the problem of the load is applied to the surface of the crack, which is in the material.

A diagram for solving these problems is shown in Fig. 1.

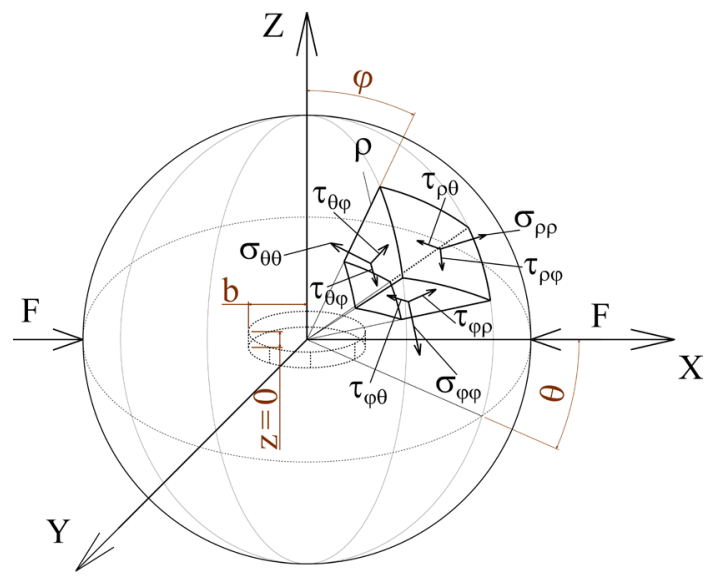

Fig 1. Scheme for determining the stresses and deformations of a material in the presence of a crack

The equations for the displacement functions in expanded form are written as follows [19]:

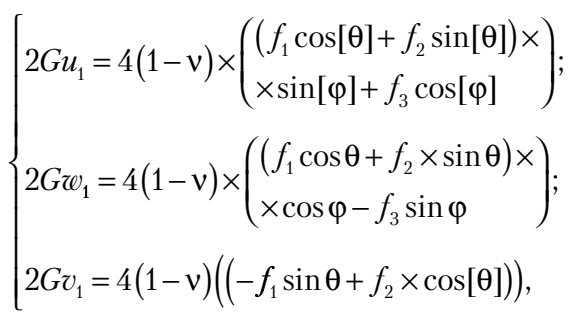

where $f_{1}, f_{2}, f_{3}$ - arbitrary harmonic functions; $G$ - shear modulus; $v$ - Poisson's ratio.

The solution of these equations is reduced to finding three harmonic functions $f_{1}, f_{2}, f_{3}$. As these functions, volumetric spherical functions are taken, which have the following form:

$$
\begin{aligned}
& f_{k}=\sum\left(A_{n} \times \rho^{n}+B_{n} \times \rho^{-n-1}\right) \times \\
& \times\left(\begin{array}{l}
C_{m} \cos [m \times \theta]+ \\
+D_{m} \times \sin [m \times \theta]
\end{array}\right) \times\left(\begin{array}{l}
L_{n m} \times P_{n m}(\mu)+ \\
+F_{n m} \times Q_{n m}(\mu)
\end{array}\right),
\end{aligned}
$$


$\mu=\cos [\varphi]-$ module; $P_{n m}(\mu)-$ Legendre function of the first kind, degree n and order m; $Q_{n m}(\mu)$ - Legendre function of the second kind, degree $\mathrm{n}$ and order $\mathrm{m} ; Q_{n}(\mu)$ - Legendre function of the second kind; $P_{n}(\mu)$ - Legendre function of the first kind; $A_{n}, B_{n}, C_{m}, D_{m}, L_{n, m}, F_{n, m}-$ constants.

For calculations it was accepted $n 1=4, m 1=1 ; n 2=5$, $m 2=2 ; n 3=6, m 3=3,1<\mu<1$. Thus, the corresponding equations for displacements and stresses were obtained. Then equation (2) will be written as follows:

$$
\begin{aligned}
& \chi_{1(m 1, n 1)}=-\frac{\left(\rho^{9} A_{4}+B_{4}\right)}{12 \sqrt{1-\mu^{2}} \rho^{5}} \theta_{1} \times \\
& \times\left(\begin{array}{l}
\left.\left(b_{1}+15 a_{1}\left(-3+7 \mu^{2}\right) \log \left[\frac{1+\mu}{1-\mu}\right]\right) F_{4,1}+\right) \\
+30 a_{1}\left(-3+7 \mu^{2}\right) L_{4,1}
\end{array}\right) \\
& \chi_{2(m 2, n 2)}=-\frac{\left(\rho^{11} A_{5}+B_{5}\right)}{4\left(-1+\mu^{2}\right) \rho^{6}} \theta_{2} \times \\
& \times\left(\begin{array}{l}
\left(b_{2}+105 a_{2}^{2}\left(-1+3 \mu^{2}\right) \log \left[\frac{1+\mu}{1-\mu}\right]\right) F_{5,2}+ \\
+210 a_{2}^{2}\left(-1+3 \mu^{2}\right) L_{5,2}
\end{array}\right) ; \\
& \chi_{3(m 3, n 3)}=-\frac{\left(\rho^{13} A_{6}+B_{6}\right)}{4\left(1-\mu^{2}\right)^{3 / 2} \rho^{7}} \theta_{3} \times \\
& \times\left(\begin{array}{l}
\left.\left(b_{3}+315 a_{3}^{3}\left(-3+11 \mu^{2}\right) \log \left[\frac{1+\mu}{1-\mu}\right]\right) F_{6,3}+\right) \\
+630 a_{3}^{3}\left(-3+11 \mu^{2}\right) L_{6,3}
\end{array}\right)
\end{aligned}
$$

where $\theta_{m}=\left(\sin [m \theta] D_{m}+\cos [m \theta] C_{m}\right)-$ coefficient depending on the change in the azimuthal angle of rotation;

$$
\begin{aligned}
& a_{k}=\mu\left(-1+\mu^{2}\right)^{k}, \\
& b_{1}=\left(-32+230 \mu^{2}-210 \mu^{4}\right), \\
& b_{2}=64-686 \mu^{2}+1260 \mu^{4}-630 \mu^{6}, \\
& b_{3}=\left(-512+7326 \mu^{2}-20286 \mu^{4}+20370 \mu^{6}-6930 \mu^{8}\right)
\end{aligned}
$$

- coefficients;

After substituting the system of equations (3) into (1), let's obtain the corresponding equations for the displacements. Knowing the displacements by a similar substitution method, let's find the stresses. The equations for displacements and stresses are not given due to their large volume.

The next step is to consider a simplified spatial problem of the destruction of a body in the presence of a crack. An ellipsoid is taken as the shape of the crack. In this form, by changing the ellipticity, it is possible to realize various degrees of curvature of the crack boundary (Fig. 1).

In this case, the boundary conditions have the following form: $\sigma_{z}=-F, \xi=0, \omega=0, \kappa=0$, where $\xi=$ const is a family of ellipsoids; $\kappa$ - hyperboloids with another cavity; $w$ - displacements in the direction of the $z$ axis; $\sigma_{z}$ - stress in the direction of the $z$ axis.

Based on mathematical transformations, displacements from the action of normal forces at any point of the body in spherical coordinates were obtained:

$$
\left\{\begin{array}{l}
u_{n}=\frac{A}{3 r^{7}}\left(\begin{array}{l}
21 r^{5}-70 r^{4} \rho+105 r^{3} \rho^{2}- \\
-84 r^{2} \rho^{3}+35 r \rho^{4}-6 \rho^{5}
\end{array}\right) \times \\
\times(-3+(-5+4 v) \cos [2 \varphi])+O[\rho-r]^{6} ; \\
v_{n}=0 \\
w_{n}=-\frac{2 A}{3 r^{7}}(-1+2 v) \times \\
\times\left(\begin{array}{l}
21 r^{5}-70 r^{4} \rho+105 r^{3} \rho^{2}- \\
-84 r^{2} \rho^{3}+35 r \rho^{4}-6 \rho^{5}
\end{array}\right) \sin [2 \varphi]+O[\rho-r]^{6},
\end{array}\right.
$$

where $r$ - radius of the sphere; $\rho$ - polar radius, the coordinate of the stress point of which it is necessary to know; $\varphi$ - zenith angle; $O[\rho-r]^{6}$ - members of a number of higher orders; $A=-a b^{2} F / 4 \mu E(m)-$ constant;

$$
E(m)=\int_{0}^{\frac{\pi}{2}} \sqrt{1-m^{2}(\sin [\varphi])^{2}} \mathrm{~d} \varphi
$$

- complete elliptic integral of the second kind; $\varphi=\pi / 2-$ amplitude of the elliptic integral in the Legendre form; $m=\sin [\alpha]-$ modulus of the integral; $\alpha$ - modular angle.

When solving the problem, let's assume that the displacement in the direction of the azimuthal angle $\theta$ is equal to 0 . That is, the stress and displacement in the body with a crack do not change with respect to the angle $\theta$.

Thus, knowing all the components of stresses and displacements for two states of the material, it is possible to calculate their total values and determine the total energy of destruction in the presence of a crack in the body.

On the basis of the equations of the minimum potential energy of a deformed body [20] with the use of strain components [18], it is possible to determine the energy that is spent on fracture. Thus, it is possible to proceed to the determination of energy consumption for the process as a whole.

The total energy consumption in the destruction of a solid body of radius $r$ in the presence of a microcrack in it is equal to the ratio of the form [21]:

$$
\begin{aligned}
& E=\frac{n^{2} b^{2} G}{4 \pi(1-v)} \ln \left(\frac{2 r}{x}\right)+4 \gamma x- \\
& -\frac{\pi(1-v)\left(\sigma_{n}^{2}+\tau_{s}^{2}\right) x^{2}}{2 G}-n b \sigma_{n} x \sin \theta,
\end{aligned}
$$

where $\sigma_{n}$ and $\tau_{s}$ - components of normal and shear stresses in the crack plane; $\theta$ - angle between the slip plane and the crack; v - Poisson's ratio; $G$ - shear modulus. The first term in (5) reflects the elastic energy of the edge dislocation; the second is the surface energy of the crack; the third is the elastic deformation energy of a solid with a crack; the fourth is the work of external forces to increase the volume of the crack during its opening [22].

When using impact destruction, in contrast to the considered approaches to the process of energy intensity of crushing, the method of physical analogies is used [23], on the basis of which the following dependence is recommended to determine the overall work:

$$
A=\frac{K \sigma_{p}^{1,5} P_{k}^{0,5}(1-v)^{2,5}(1+v) S^{b} n^{c}}{v^{1,5} E_{M}},
$$

where $K$ - coefficient that takes into account the conversion of shock load into forces that breaks the material; $P_{k}-$ contact 
strength of the rock; $S$ - area over which the material is split, $\mathrm{m}^{2}$; $n$ - the number of blows to destruction; $b$ and $c$-indicators characterizing the scale effect and the effect of fatigue, respectively, which depend on the structure and strength characteristics of rocks. All indicators of this equation are valid only when a load is applied at a speed of $v \ll 7-13 \mathrm{~m} / \mathrm{s}$.

Of course, the indicated hypotheses (5), (6) make it possible to determine the useful energy, which is spent only on the process of material destruction. Turning to real crushing systems, it is necessary to additionally take into account the energy consumption for internal supports in the system and its heat dissipation [24].

In general, the total energy consumed by crushers for the process of crushing the material will have the following form [25]:

$$
E_{\text {tot }}=T+P,
$$

where $T$ - kinetic energy of the movement of the masses of the crusher; $P$ - potential energy of deformation of the springs.

\section{Results of the study of energy costs for the process of material destruction in the crushing chamber of a vibratory jaw crusher}

5.1. Research and analysis of changes in the main parameters affecting the zones to ensure the reduction of energy consumption of the vibration crusher

The study of stresses and strains in a material during its crushing [18] opens up the possibility of calculating their total values and determining the total energy of destruction in the presence of a crack in the body.

Based on the superposition of equations for determining the displacements of a body without breaking the integrity (2) and equations for determining the displacements of a body with a crack (4), the corresponding graphs were constructed. The graphs of the dependence of the principal stresses on the size of the major semiaxis of the crack ellipse are shown in Fig. 2. The graph of stress versus zenith angle $\varphi$ is shown in Fig. 3. The change in stress $\sigma_{\rho}$ depending on the radius $\mathrm{r}$ is shown in Fig. 4.

As follows from the graphs (Fig. 2), the dependences of stresses on the size of the major semiaxis of the ellipse have a linear characteristic within the framework of the calculations, the numerical values of which were used to determine the parameters of the crusher.

A significant effect of the angle of application of force on the change of stresses is observed in the range of $0.3-0.5$ (Fig. 3).

A more complex law of variation of normal stresses $\sigma_{\rho}$ depending on the radius $r$ is noted within $9-12 \mathrm{~mm}$, Fig. 4.

When crushing material, the working cycle of oscillation of the crushing jaws of a vibrating jaw crusher can be conditionally divided into 3 stages:

1) leaving the crushing jaw and advancing the material in the direction of its unloading due to the forces of gravity;

2) convergence of the crushing jaw and the material, due to which external zones of destruction are formed and internal stresses accumulate;

3) further movement of the stripping cheeks, compression of the material and an increase in internal stresses, as a result of which the destruction of the material occurs. Thus, in some approximation, a static model can be taken as a model of the destruction process.
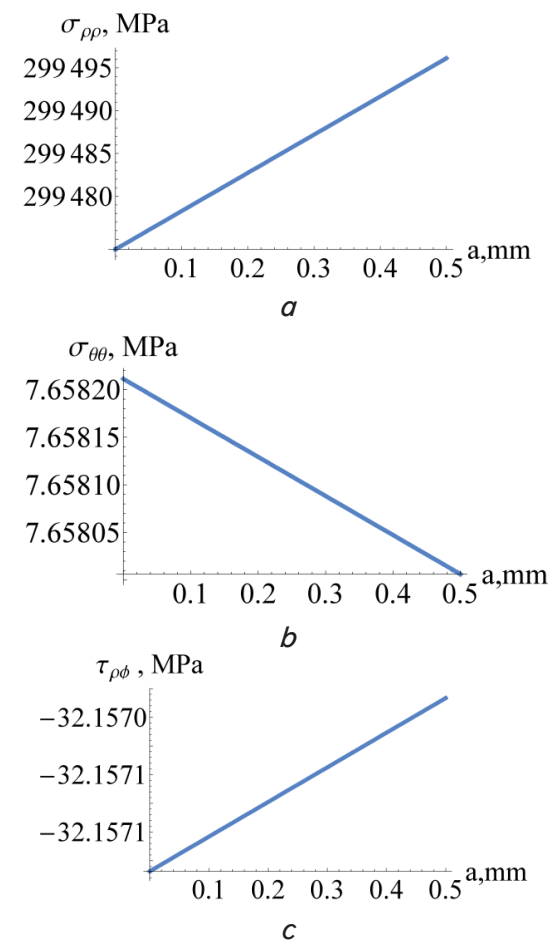

Fig. 2. Graph of stress dependence on the size of the major semiaxis of the ellipse: $a-\sigma_{\rho}-a ; b-\sigma_{\theta}-a ; c-\tau_{\rho \varphi}-a$

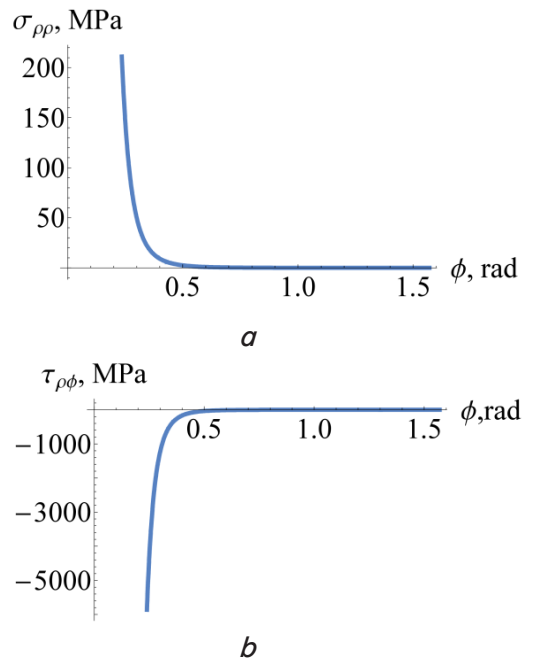

Fig. 3. Graph of stress change depending on the angle of application of force $\varphi: a-\sigma_{\rho}-\varphi ; b-\tau_{\rho \varphi}-\varphi$

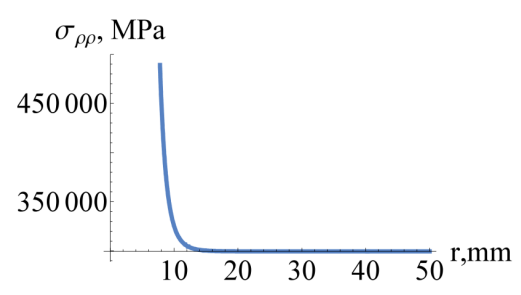

Fig. 4. Graph of changes in normal stresses $\sigma_{\rho}$ depending on the radius $r$

5. 2. Determination of modes and rational parameters to ensure a decrease in energy consumption and an increase in the efficiency of the technological process of material grinding

Consider two vibration-driven crushing systems shown in Fig. 5. The vibrating jaw crusher (Fig. 5, $a$ ) is made in the 
form of a three mass resonance system, in which rectilinear vibrations are realized and the dynamics of which occurs in a three-mass scheme. The vibration exciter 1 with the base plate is the first or active mass, the moving body 5 is the second mass, the middle plate with the inner armor 4 is the third mass. All masses of the crusher are connected in pairs by elastic systems 2 and 3. Vibration cone crusher (Fig. 5, $b$ ) consists of a vibration exciter 1, mounted on a stationary cone 7 of the crusher. The fixed cone 7 is connected to the crusher body 4 through the adjusting ring 6 and the elastic system 2 . The crusher body 4 is installed on the foundation through the elastic system 3 . The movable crusher cone is driven through the drive 5 .

The energy consumed by the electric motors of the crushers (Fig. $5, a, b$ ) is spent on performing work on crushing the material. Additionally, energy costs are spent on deformation of the elastic system, overcoming friction forces, heat losses, deformation of crusher parts and other losses. However, the greatest energy consumption during the operation of crushers goes into the kinetic energy of the crushing plates and the potential energy of deformation of the springs.

$$
\begin{aligned}
& E_{t o t}=\left[\frac{1}{2} m_{r c} \times\left(v_{x}{ }^{2}+v_{y}{ }^{2}\right)+\frac{I_{r c} \times \dot{\varphi}^{2}}{2}+\frac{1}{2} m_{s c} v_{s c}^{2}\right]+ \\
& +\left[\frac{c_{d r}\left(n \times x_{1}\right)^{2}}{2}+\frac{c_{1} x_{1}{ }^{2}}{2}\right],
\end{aligned}
$$

where

$$
T_{1}=\frac{1}{2} m_{r c} \times\left(v_{x}^{2}+v_{y}^{2}\right)+\frac{I_{r c} \times \dot{\varphi}^{2}}{2}
$$

- kinetic energy of the rolling of the moving cone [7];

$$
T_{2}=\frac{1}{2} m_{s c} v_{s c}^{2}
$$

- kinetic energy of vibrations of a stationary cone; $m_{r c}$ is the mass of the movable cone; $I_{r c}$ - the central moment of inertia of the cone; $x_{1}, y_{1}$ - Cartesian coordinates of the center of the cone; $\varphi$ - angle of rotation of the movable cone around
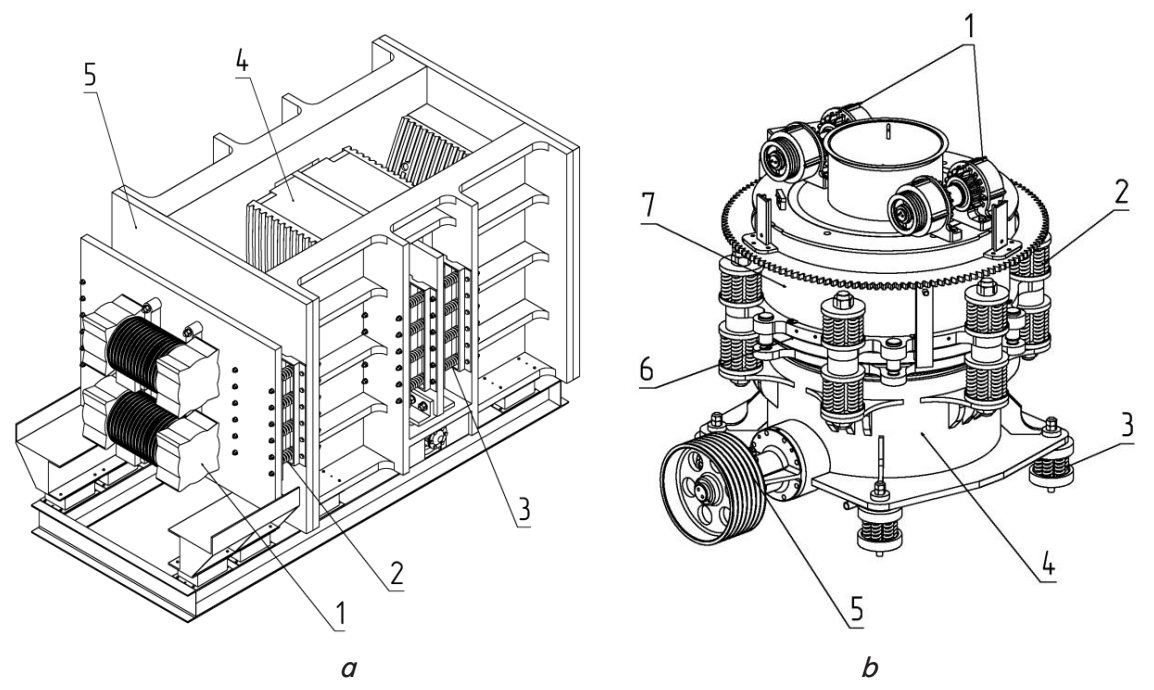

Fig. 5. Design diagram of vibrating crushers: $a$ - vibrating jaw crusher with three vibrating masses; $b$ - cone crusher with vibrators on the body its axis; $m_{s c}-$ total mass of the stationary cone; $v_{s c}$ - speed of vertical displacements of a stationary cone; $c_{d r}$ - material stiffness coefficient; $c_{1}$ - stiffness coefficient of the elastic system that connects the stationary cone and the crusher body.

Sandstone with a tensile strength of up to $20 \mathrm{MPa}$ was taken as a model of the material of destruction.

Based on the equations of motion of a vibratory jaw crusher [25], the speeds of movement of the vibrational masses of the crusher were determined numerically, which are shown in Fig. 5.

Regarding graph 6 , it should be noted that these velocities of mass fluctuations are valid under the conditions of the disturbing force frequency $f=19 \mathrm{~Hz}$ and the total mass of the laboratory setup $m_{\text {tot }}=334 \mathrm{~kg}$.

Based on the general equation of energy consumption for a vibratory jaw crusher with three vibrational masses (7), the following equation was derived:

$$
E_{\text {tot }}=\left[\sum_{n=1}^{3} \frac{m_{n} \dot{x}_{n}^{2}}{2}\right]+\left[\frac{1}{2}\left(\begin{array}{l}
c_{f} x_{2}^{2}+ \\
+c_{1}\left(3 x_{1}^{2}-4 x_{1} x_{2}+2 x_{2}^{2}\right)+ \\
+4 c_{2}\left(x_{2}-x_{3}\right)^{2}
\end{array}\right)\right],
$$

where $m_{n}$ - respectively, the $n$-mass of the crusher, kg; $c_{1}, c_{2}$ - stiffness of the springs connecting the masses of the crusher; $x_{1}, x_{2}$ and $x_{3}$ - the corresponding displacements of the masses of the crusher, $\mathrm{mm} ; c_{f}$ - the stiffness of the connections of the system that connects the machine to the foundation.

When determining the total energy of a vibratory cone crusher (Fig. 5, b), let's assume that the movable cone is an absolutely rigid cylinder that rolls over a solid undeformed body, which has the shape of an ellipse. In turn, let's assume that the crusher body with vibrators is a solid body with a mass $m_{t o t}$ and a moment of inertia I, fixed on a fixed base by springs with a stiffness of the $c_{i s}$ :

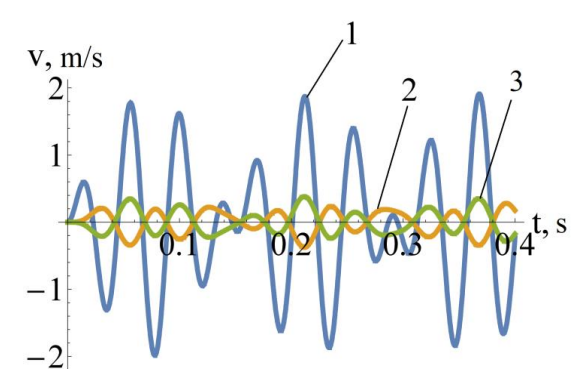

Fig. 6. The speed of movement of the masses of the vibrating jaw crusher: 1 - first mass;

2 - second mass; 3 - third mass

Using the first part of Eq. (4) and the equations of motion of the masses of the vibratory jaw crusher [25], graphs of the kinetic energy consumption were constructed, which is spent on moving the vibrational masses of the vibratory crusher Fig. 7.

From the graphs in Fig. 7, it is clear that the total kinetic energy of mass motion is within $120 \mathrm{~J}$. However, equation (8), 
which were used to plot the graphs in Fig. 6, do not take into account the losses due to the forces of dry and viscous friction.
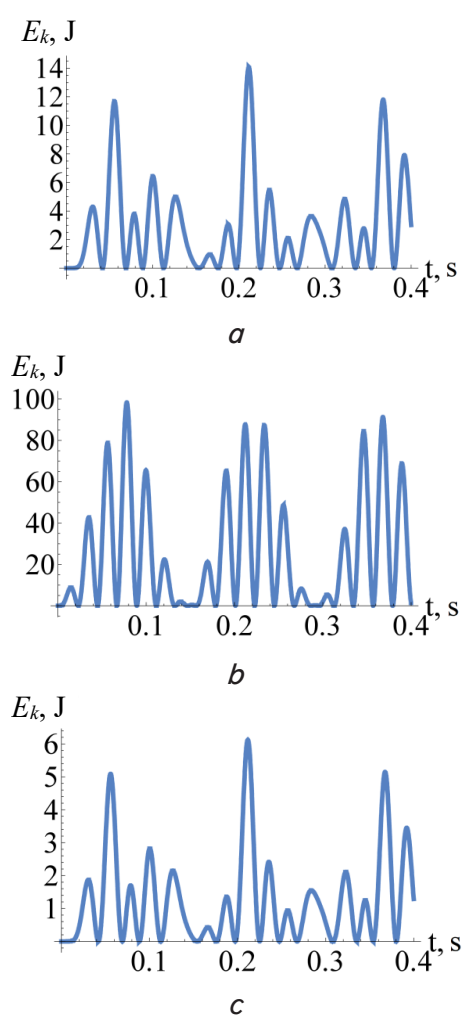

Fig. 7. Graph of changes in the kinetic energy of vibrational masses of a vibrating jaw crusher: $a$ - first mass; $b$ - second mass; $c$ - third mass

To build graphs of potential energy consumption for the crushing process, it is necessary to find the displacement of the vibrational masses.

The equations of motion of a vibratory shock crusher are as follows [25]:

$$
\left\{\begin{array}{l}
m_{1} \ddot{x}_{1}+2 x_{1} c_{1}-x_{2} c_{1}=F_{0} \sin \omega t ; \\
\left(m_{2}+k m_{m}\right) \ddot{x}_{2}+x_{2} c_{1}-x_{1} c_{1}+ \\
+2 c_{2} x_{2}-2 c_{2} x_{3}+x_{2} c_{o p}=0 \\
\left(m_{3}+k m_{m}\right) \ddot{x}_{3}+2 c_{2} x_{3}-2 c_{2} x_{2}=0 \\
F_{d r} \leq c_{2} x_{n} \\
x_{1}>0 ; x_{3}>0 ; x_{2}<0 \\
x_{2}+x_{3}=\xi^{*} D_{\max } .
\end{array}\right.
$$

The displacement of the vibrational masses of the crusher, determined by the numerical method based on the equation of motion, is shown in Fig. 8.

The graph of Fig. 8 shows that the system setting is correct, i. e. the second and third masses oscillate in antiphase. The total amplitude of movement of the second and third oscillating masses is in the range of $8-10 \mathrm{~mm}$. The minimum required value of the oscillation amplitude of the crushing plates of the vibrating crusher, at which the failure occurs, is in the range of $3-5 \mathrm{~mm}$.

The graph of the total potential energy consumption for the destruction process in the crushing chamber of the vibrating jaw crusher is shown in Fig. 9. This graph was constructed numerically using equations (8) and (10).

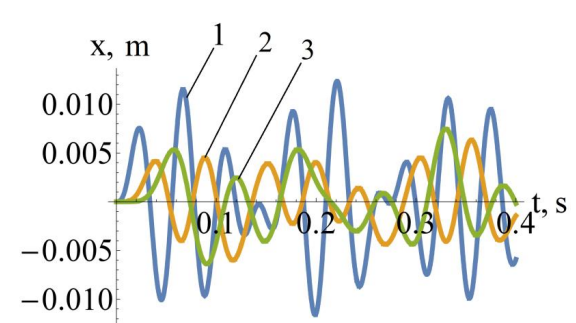

Fig. 8. Changing the amplitude of the oscillating masses of the vibrating jaw crusher: 1 - the first mass;

2 - another table; 3 - third table

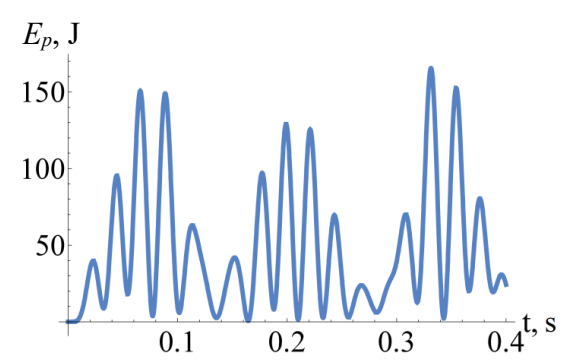

Fig. 9. Graph of potential energy change of vibrating jaw crusher

Graphs in Fig. 7 and Fig. 9 indicate insignificant energy consumption for the destruction process. It should be noted here that the system is tuned to a resonant mode of operation. That is, the graphs in Fig. 6 and Fig. 8 do not show the energy consumption for the system to enter the resonance mode and to maintain its operation in this mode. In addition, it should be borne in mind that for the sake of simplicity, the equations of motion [25] do not take into account the dissipation of energy.

To assess the energy of destruction of a body containing a crack, graphs of the instantaneous force were plotted, which occurs when the crushing plates of the second and third masses of the vibration crusher collide with the material in Fig. 10. Equations (8) and (10) were used to construct this graph.

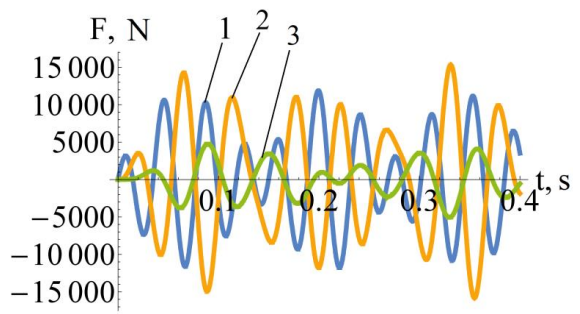

Fig. 10. The value of the instantaneous force when the vibrational masses of the vibrating jaw crusher move:

1 - first mass; 2 - second mass; 3 - third mass

The given graph (Fig. 10) testifies to the adequacy of the calculation scheme to the accepted prerequisites. So, the first mass moves in antiphase with the second mass. The third mass, accumulating energy, is an auxiliary source of energy transfer to the material crushing process.

In equation (4), to determine the energy consumption of a vibratory jaw crusher, the fracture resistance of the material is considered as the added mass. That is, the indicator of the stiffness of the material is the parameter that includes energy consumption at the micro level. In equation (5) for a vibratory cone crusher, this parameter is directly the material stiffness coefficient. Thus, the obtained analytical 
dependences (4), (5) reveal the physical essence of the grinding process, take into account the conditions of interaction between the crusher and the material to be crushed. The practical application of the obtained dependences requires the numerical values of the characteristics of a particular material, which is the subject of further research.

\section{Discussion of the results of studying the energy characteristics of material destruction in the crushing chamber of the crusher}

The studies carried out and their results indicate that the main stresses that cause the destruction of the material are the displacement stress. With an increase in the size of the major semiaxis of the crack ellipse, the total stresses decrease, which indicates the destruction of a brittle body. However, the stress $\sigma_{\rho}$ acting along the radius slightly increases. Graphs (Fig. 3) reflect the picture of stress distribution in a spherical body relative to the point of application of the force along the angle $\varphi$. The graph (Fig. 4) shows the change in normal stresses depending on the distance $r$. Analysis of the graph (Fig. 4) makes it possible to conclude that at $r->0$, the stress grows indefinitely. As a result, stress and strain in a cracked body must be determined in the vicinity of the center of the body. In addition, it was found that the complete elliptical integral of the second kind has an insignificant effect on the change in the stress pattern in the body. It should be noted that when solving the problem using the Legendre functions, 15 constants appear in the stress equations, which in total have a significant effect on the stress pattern in the body. These constants were found by a numerical method based on the equations of the potential energy of a deformed body.
Equations (4) and (5) fully reflect the picture of energy costs, since they include energy costs for the movement of the crusher links and costs for material destruction. Such studies are planned as a continuation of the topic under consideration through experimental studies and consideration of several members of the series and optimization of the parameters of crushing machines. The proposed approach for studying the energy characteristics of material destruction in the crusher chamber with a guaranteed zone of stability of parameters in the resonance zone can be used for other processes. Such processes include the destruction of materials during dynamic cutting of soils, processing in mills and sorting of materials, which are widely used in various European countries.

\section{Conclusions}

1. The change in the amplitude of vibrational masses of a vibrating jaw crusher is investigated: the main parameters affecting the zones of ensuring a decrease in energy consumption of a vibratory crusher. The total amplitude of displacement of the second and third vibrational masses is in the range of $8-10 \mathrm{~mm}$. The condition for tuning the system to the required mode is provided, that is, the second and third masses oscillate in antiphase.

2. It has been determined that the minimum required value of the vibration amplitude of the crushing plates of a vibration crusher, at which destruction occurs, is within $3-5 \mathrm{~mm}$. The greatest energy consumption during the operation of crushers goes into the kinetic energy of the crushing plates and the potential energy of deformation of the springs.

\section{References}

1. Nguyen, T. N., Kolenko, G. S. (2020). Analysis of the fracture mechanics and workability of a gas turbine blade in the presence of a crack. Materials Science. Power Engineering, 26 (3), 56-69. doi: https://doi.org/10.18721/JEST.26304

2. Terentiev, O., Streltsova, I. (2015). Energy intensity and specific surface energy of rock breaking by magnetic hydrocavitation stressing. Visnyk NTUU «KPI». Seriya «Hirnytstvo», 28, 29-35.

3. Vasiliev, L. M., Vasiliev, D. L., Malich, M. G. (2021). Modeling the process of disintegration of solid materials by asymmetric loading in crushing machines in order to find ways to reduce energy costs. Energy- and resource-saving technologies of developing the raw-material base of mining regions, 457-473. doi: https://doi.org/10.31713/m1028

4. Hong, S. J., Yang, H. J. (2019). A Study on the Impact Load Quantification of the Jaw Crusher. Journal of Drive and Control, 16 (2), 1-7. doi: https://doi.org/10.7839/KSFC.2019.16.2.001

5. Pothina, R., Kecojevic, V., Klima, M. S., Komljenovic, D. (2007). Gyratory crusher model and impact parameters related to energy consumption. Mining, Metallurgy \& Exploration, 24 (3), 170-180. doi: https://doi.org/10.1007/bf03403212

6. Sokur, M., Biletskyi, V., Sokur, L., Bozhyk, D., Sokur, I. (2016). Investigation of the process of crushing solid materials in the centrifugal disintegrators. Eastern-European Journal of Enterprise Technologies, 3 (7 (81)), 34. doi: https://doi.org/10.15587/17294061.2016 .71983

7. Fladvad, M., Onnela, T. (2020). Influence of jaw crusher parameters on the quality of primary crushed aggregates. Minerals Engineering, 151, 106338. doi: https://doi.org/10.1016/j.mineng.2020.106338

8. Lapin, R., Kuzkin, V. (2019). Calculation of the normal and shear compliances of a three-dimensional crack taking into account the contact between the crack surfaces. Letters on Materials, 9 (2), 234-238. doi: https://doi.org/10.22226/2410-3535-2019-2-234-238

9. Zou, J., Han, J., Yang, W. (2020). Investigating the Influences of Indentation Hardness and Brittleness of Rock-Like Material on Its Mechanical Crushing Behaviors. Mathematical Problems in Engineering, 2020, 1-16. doi: https://doi.org/10.1155/2020/4713532

10. Beloglazov, I. I., Yusupov, G. A., Stepanyan, A. S., Feoktistov, A. Y. (2018). Disintegration process modeling for a jaw crusher with complex jaws swing. Obogashchenie Rud, 2, 3-8. doi: https://doi.org/10.17580/or.2018.02.01

11. Gorobets, L. J., Fedoskina, E. V., Verhorobina, I. V. (2017). Effects of dynamic quality of ladening of geterogen material at crushing. Heotekhnichna mekhanika, 137, 93-106. Available at: http://dspace.nbuv.gov.ua/handle/123456789/158641 
12. Nesterenko, M., Nazarenko, I., Molchanov, P. (2018). Cassette Installation with Active Working Body in the Separating Partition. International Journal of Engineering \& Technology, 7 (3.2), 265. doi: https://doi.org/10.14419/ijet.v7i3.2.14417

13. Bernyk, I., Luhovskyi, O., Nazarenko, I. (2018). Effect of rheological properties of materials on their treatment with ultrasonic cavitation. Materiali in Tehnologije, 52 (4), 465-468. doi: https://doi.org/10.17222/mit.2017.021

14. Nazarenko, I., Dedov, O., Bernyk, I., Rogovskii, I., Bondarenko, A., Zapryvoda, A. et. al. (2020). Determining the regions of stability in the motion regimes and parameters of vibratory machines for different technological purposes. Eastern-European Journal of Enterprise Technologies, 6 (7 (108)), 71-79. doi: https://doi.org/10.15587/1729-4061.2020.217747

15. Perelman, G. (2002). The entropy formula for the Ricci flow and its geometric applications. arXiv.org. Available at: https://arxiv. org/pdf/math/0211159.pdf

16. Morgan, J. W., Tian, G. (2007). Ricci flow and the Poincare conjecture. arXiv.org. Available at: https://arxiv.org/pdf/math/0607607.pdf

17. Ciężkowski, P., Maciejewski, J., Bąk, S. (2017). Analysis of Energy Consumption of Crushing Processes - Comparison of One-Stage and Two-Stage Processes. Studia Geotechnica et Mechanica, 39 (2), 17-24. doi: https://doi.org/10.1515/sgem-2017-0012

18. Nazarenko, I., Mishchuk, E. (2014). Research process of destruction of the material in the grinding chamber of the vibrating jaw crusher. Hirnychi, budivelni, dorozhni ta melioratyvni mashyny, 84, 55-63.

19. Mozharovskyi, M. S. (2002). Teoriya pruzhnosti, plastychnosti i povzuchosti. Kyiv: Vyshcha shkola, 308.

20. Khalilpour, S., BaniAsad, E., Dehestani, M. (2019). A review on concrete fracture energy and effective parameters. Cement and Concrete Research, 120, 294-321. doi: https://doi.org/10.1016/j.cemconres.2019.03.013

21. Levin, V. A., Morozov, E. M., Matvienko, Yu. G. (2004). Izbrannye nelineynye zadachi mekhaniki razrusheniya. Moscow: FIZMATLIT, 408.

22. Munoz, H., Taheri, A., Chanda, E. K. (2016). Fracture Energy-Based Brittleness Index Development and Brittleness Quantification by Pre-peak Strength Parameters in Rock Uniaxial Compression. Rock Mechanics and Rock Engineering, 49 (12), 4587-4606. doi: https://doi.org/10.1007/s00603-016-1071-4

23. Johansson, M., Bengtsson, M., Evertsson, M., Hulthén, E. (2017). A fundamental model of an industrial-scale jaw crusher. Minerals Engineering, 105, 69-78. doi: https://doi.org/10.1016/j.mineng.2017.01.012

24. Liu, R., Shi, B., Li, G., Yu, H. (2018). Influence of Operating Conditions and Crushing Chamber on Energy Consumption of Cone Crusher. Energies, 11 (5), 1102. doi: https://doi.org/10.3390/en11051102

25. Mischuk, Y., Nazarenko, I. (2019). Research of the dynamics of a vibratory jaw crusher of bilateral action. Girnichi, Budivelni, Dorozhni Ta Meliorativni Mashini, 94, 5-15. doi: https://doi.org/10.32347/gbdmm2019.94.0101 